\title{
LA FRANCE, UNE PASSION ÉGYPTIENNE. UNE LECTURE DU TARBOUCHE DE ROBERT SOLÉ
}

\author{
Manal HosNY \\ Université de Damnhour, Egypte \\ GUST, Koweït
}

\begin{abstract}
En): According to Robert Solé in L'Égypte passion française, Egypt used to be "one of the most lively francophone communities of the planet. "The writer, born in Cairo into a Levantine family with whom he left Egypt when he was 17 years old, is well versed in such a topic. His first novel Le Tarbouche takes place in the country of his childhood. This family saga that recalls the history of the Greek Catholic community established in Egypt since the $19^{\text {th }}$ Century, evokes the questions of belonging and identity. It also highlights the protagonists' relation to languages: Arabic, the language of their origin which they do not speak well and French, the language they favor and adopt as their "mother tongue". Our aim in the following study is to focus on identity; first, we wish to discuss the infiltration of the French language and culture into different spheres of life in Egypt and second, we plan to analyse the correlation between this linguistic and cultural phenomenon and the sense of identity of the main characters.
\end{abstract}

Résumé (Fr) : L'Égypte fut «l'une des communautés francophones les plus vivantes de la planète » écrit Robert Solé dans L'Égypte, passion française, sous-entendant que la France fut à son tour une passion égyptienne. Né au Caire et issu d'une famille levantine, l'écrivain qui, à dix-sept ans, quitte l'Égypte avec sa famille, est bien placé pour traiter du sujet. Son premier roman, Le Tarbouche, se passe justement dans le pays de son enfance. Cette saga familiale, tout en restituant l'histoire de la communauté gréco-catholique, soulève le problème de l'appartenance et de l'identité. Les interrogations identitaires des personnages passent le plus souvent par le biais de leur rapport à la langue, ou plus précisément aux langues : l'arabe leur langue d'origine qu'ils maîtrisent mal et le français leur langue d'adoption à laquelle ils s'affectionnent et qui leur sert de langue « maternelle ».

Dans cette étude, nous nous penchons donc sur cette œuvre pour cerner, dans un premier temps, l'infiltration de la langue et de la culture françaises dans les différents domaines de la vie en Égypte, avant d'analyser, dans un deuxième temps, la corrélation entre ce phénomène à la fois linguistique et culturel et la conscience identitaire des personnages.

Keywords : Robert Solé; Egypt; Identity; minorities; exile; acculturation.

Mots-clés : Robert Solé ; Égypte ; identité ; minorités ; exil ; acculturation.

Pour certains, associer la francophonie à un ancien protectorat britannique comme l'Égypte peut sembler paradoxal. Pourtant, dès la fin du XIX ${ }^{\mathrm{e}}$ siècle, ce territoire politiquement contrôlé par les Anglais devient une zone d'hégémonie culturelle française. La langue et la culture françaises s'implantent avec force dans le pays si bien que la première moitié du $\mathrm{XX}^{\mathrm{e}}$ siècle marque l'âge d'or de cette société orientale francophone. L'Égypte devient, selon Robert Solé, l'une des communautés francophones les plus vivantes de la planète. En fait, l'auteur de L'Égypte, passion française (1997) nous semble bien placé pour traiter du sujet. Né au Caire dans une famille levantine, francophone de par sa formation et Français de par son installation définitive en France, Solé quitte l'Égypte avec les siens à l'âge de dix-sept ans. Son premier roman, Le Tarbouche (1992), se passe justement dans le pays de son enfance. 
Saga familiale, retraçant la trajectoire des Batrakani depuis leur arrivée en Égypte dans des circonstances tragiques jusqu'à leur dispersion et leur départ vers d'autres exils, Le Tarbouche met en scène plusieurs autres familles syriennes dont les destins se croisent et s'entrelacent au grès des péripéties, comme les Touta, les Ayrout et les Yared. L'auteur nous introduit ainsi dans l'intimité de cette communauté levantine à cheval entre deux mondes, tenant à la fois de l'Orient et de l'Occident. Évoluant entre les quartiers du Caire, d'Héliopolis et d'Alexandrie, le roman restitue une Égypte du temps des rois, à la fois tragique et extravagante, une Égypte prise entre un cosmopolitisme affairiste et une exigence nationaliste, une Égypte à jamais disparue.

En fait, ce roman du temps passé est un tribut payé à la mémoire. Cependant, plutôt qu'une reconstitution linéaire des actions, l'auteur effectue un montage anachronique des faits. Le récit commence par un prologue où le narrateur, un certain Charles, nous fait part de sa naissance et se clôt, en guise de prologue, par un fragment du journal de son oncle Michel rédigé en exil. Si le début du roman nous suggère que c'est Charles le héros de l'ouvrage, il n'en est rien. Cette histoire est la sienne parce que celle de sa famille, parce que raconter son histoire, c'est aussi raconter la vie des autres. Il remonte ainsi dans le temps pour nous relater l'ascension de son grand-père Georges Batrakani et effectue un va-et-vient continu qui nous prend de 1916 à 1903, de 1919 à 1890 et de 1925 à 1942 pour se terminer en 1963.

Dans cette étude, nous nous pencherons sur Le Tarbouche pour cerner, dans un premier temps l'infiltration de la langue et de la culture françaises dans les différents domaines de la vie égyptienne, avant d'analyser, dans un deuxième temps, la corrélation entre ce phénomène à la fois linguistique et culturel et la conscience identitaire des personnages.

En effet, s'inspirant de son expérience personnelle comme de celle de son entourage, l'auteur évoque les différentes nuances de ce métissage culturel qui marque profondément la bourgeoisie égyptienne. Avec humour, le narrateur nous dévoile les circonstances de son premier contact avec la France. S'adressant au gynécologue français qui l'a mis au monde, il déclare :

Et nous, là-dedans, les Rafik, Nabil, Pierre ou Charles? Songez, monsieur, que vous étiez notre premier contact physique avec le monde extérieur. Dès que nous pointions le nez ou le derrière, c'étaient des mains françaises qui nous tiraient dehors. La France pour nous accueillir! Autant dire que la notion de mère patrie n'a jamais été très claire dans nos esprits. (p. 11)

C'est qu'en effet, la France fait l'objet d'un engouement sans pareil au sein de cette communauté levantine très francisée «sans avoir dans les veines une goutte de sang français. » (p. 11) Par tradition, même avant l'établissement du mandat français, les Syriens considèrent la France comme «leur protectrice naturelle », cette dernière, de son côté, sait qu'ils «sont sa meilleure clientèle en Égypte. » (p. 102) Une parenthèse du roman nous apprend que cette relation privilégiée remonte au temps des capitulations quand la France fut chargée de protéger les minorités chrétiennes de l'Empire ottoman. En fait, consciente de l'importance stratégique des liens entretenus avec les chrétiens en Syrie et surtout au Mont Liban, la diplomatie française étend son patronage sur ces -80 - 
chrétiens orientaux bien avant la vague d'émigration qui les emmène en Égypte. C'est donc tout naturellement que ces «sujets locaux» de la Sublime Porte apprennent le français chez les missionnaires. (ABECASSIS, $2000: 73$ )

Une fois en Égypte, cette connaissance de la langue française va devenir leur véritable atout. C'est elle qui leur ouvre la voie des carrières administratives subalternes, soit dans les sociétés fondées sur des intérêts mixtes où le français s'était depuis longtemps imposé, soit dans l'administration, comme c'est le cas d'Élias Batrakani, l'arrière-grand-père de notre personnage :

Les Anglais embauchaient. Désireux de prendre en main l'administration locale et la réformer, ils cherchent des intermédiaires assez sûrs connaissant l'arabe, mais aussi d'autres langues, et ayant un minimum deformation générale. Peu de musulmans et de coptes correspondaient à ce profil. Nous les Syriens', nous sommes pour l'Angleterre un don du ciel, constatait Élias à l'époque. Que voulez-vous, nous avons la formation. (p. 51)

Mais ce sont surtout les tribunaux mixtes fondés en 1875 et dont les compétences débordent assez vite le cadre des traits capitulaires pour s'étendre " à toutes les questions où un intérêt étranger peut ou pourrait être engagé » (L'Égypte indépendante, 1938, p. 124) qui rendent l'usage du français indispensable en Égypte. D'origine française, la législation mixte calque ses codes sur les codes français, sa jurisprudence fait de larges emprunts de dispositions à des lois françaises. C'est d'ailleurs dans cette institution vénérable que Georges commence sa carrière en tant que greffier. Voir un de ses fils exercer dans ces tribunaux, va être pour lui un signe d'ascension sociale :

Comme la plupart de ses camarades, Paul se destinait aux tribunaux mixtes où tout se plaidait en français. Cette institution prestigieuse (...) était compétente pour tout litige civil ou commercial entre desétrangers de nationalités différentes ou entre desétrangers et des Égyptiens. (p. 135)

Langue des affaires et du commerce, de l'administration et de la jurisprudence, le français devient alors « la langue du succès ». C'est sans doute la raison pour laquelle Mima Yared, la mère de Sélim, qui connait de graves problèmes d'ordre financier depuis la mort de son mari Khalil, veut donner à ses enfants une éducation française, même au prix de durs sacrifices. Elle-même, orpheline et sans ressources, avait été recueillie par les Dames du Bon Pasteur de Choubra ${ }^{2}$ qui lui apprirent à parler le français. Cependant, si le français devient la langue de scolarisation des Syriens quel que soit leur niveau économique ou social, le résultat est loin d'être toujours satisfaisant :

Sélim avait fait sa première année de scolarité au collège patriarcal. La plupart des élèves, appartenant à des familles modestes, connaissaient mal le français. Il était interdit de parler arabe en cours de récréation e les prêtres donnaient l'exemple, comme le Père Ackaoui, qui était loin de briller dans la langue de Bossuet. Ce volumineux ecclésiastique croyait bien faire en traduisant systématiquement des expressions arabes en français. (p. 162-163)

\footnotetext{
${ }^{1}$ On dirait Chawam en dialecte égyptien pour désigner vaguement les syro-libanais sans aucune distinction territoriale, le Cham correspondant à l'ancienne division de l'empire islamique et englobant la Syrie, le Liban, la Jourdanie et la Palestine.

${ }^{2}$ Un quartier du Caire.
} 
Pour atteindre un bon niveau, il faut aller dans un des établissements scolaires gérés par les différentes congrégations catholiques qui se multiplient en Égypte dès la fin du XIX ${ }^{\mathrm{e}}$ siècle, le Collège de la Salle par exemple, "chez les frères des écoles chrétiennes. Ceux-ci (...) contrairement au frère Ackaoui (...) ne risquaient pas d'employer des expressions arabes. Français, ils l'étaient jusqu'au bout de la sandale. » (p. 163-164), Mais ce sont les Jésuites du Collège de La Sainte-Famille qui forment l'élite en Égypte. Pour Georges, ancien élève du collège de La Salle "mettre ses enfants chez les jésuites avait été (...) un signe d'ascension sociale », d'ailleurs, "dans la famille de sa femme, tous les garçons fréquentaient ce collège qui était le plus cher du Caire, le plus difficile d'accès et (...) qui assurait la meilleure formation. » (p. 44)

$\mathrm{Si}$ au départ, cet établissement scolaire ne recevait que des catholiques, les congréganistes accueillirent très tôt des musulmans, des juifs, et des coptes; «leur souci de former une élite appelée à gouverner le pays, ayant pris le pas sur leur projet initial. » (ABECASSIS, $2000:$ 89)En effet, le cinquantenaire du collège réunit ministres, pachas, magistrats et autres hauts fonctionnaires, tous anciens élèves des Jésuites. "À la table du Père recteur, on ne comptait pas moins de quatre pachas. L'assistance était truffée de conseillers royaux, de hauts fonctionnaires et de magistrats, dans une ambiance de joyeuse camaraderie. » (p. 170) Ce recrutement, qui a permis la "reproduction d'une classe favorisée de fonctionnaires et de notables » (ABECASSIS, 2000: 89), impose la langue et la culture françaises et provoque la colère du clergé oriental qui voit d'un mauvais œil l'emploi du français dans l'enseignement du catéchisme, emploie qui, selon eux, confirme l'hégémonie culturelle française et facilite la latinisation des enfants. Mgr Cadi le déclare vertement à André, le fils ainé de Georges, «Vous, les jeunes, vous allez dans des collèges religieux français. Ce sont de bons collèges, je ne dis pas. Mais, on ne vous fait assister qu'à des messes latines. Vous perdez l'habitude de notre belle liturgie. Vous devenez des étrangers dans votre propre église » (p. 109). Ce dernier ne peut le contredire, puisqu'en effet, le jeune homme désire partir en France pour entrer dans la Compagnie de Jésus.

Bien plus, «nombre de familles catholiques avaient d'ores et déjà adopté le français comme première et parfois unique langue » (ABECASSIS, $2000: 87$ ) et les riches familles syriennes de rite grec-catholique comme les Touta et les Ayrout semblaient marcher sur leurs pas. Si, Georges, tout comme son gendre Sélim qui a grandi dans un quartier populaire, maitrise bien l'arabe qu'il avait parlé à la maison toute son enfance, sa femme, Yolande, "parle l'arabe comme un pied» (p. 186) et n'arrive pas à poursuivre une conversation avec la femme de Makram, l'ami copte de Georges. Lola, leur fille, "à l'âge de treize ou quatorze ans, pour retenir deux lignes en arabe, se les faisait transcrire en français » (p. 394), sa sœur Viviane est « handicapée » par son arabe et n'arrive pas à dialoguer avec Hassan, tandis que Michel connait l'arabe «suffisamment pour lire Al Ahram chaque matin.» (p. 394) En effet, l'arabe enseigné dans les écoles françaises ne retenait point l'attention des élèves qui le négligeaient impunément. Le lien entre langue maternelle, langue des parents et langue du pays que doivent démêler les immigrés semble être remodelé au profit du français : 
«Au collège du Caire, les élèves (...) considéraient [l'arabe] comme une langue étrangère et s'y intéressaient le moins possible. (...) À vrai dire, l'exemple venait de haut : le roi Fouad connaissait mal l'arabe et le Conseil des ministres se tenait généralement en français.» (p. 181) Seul André parle correctement l'arabe. L'histoire de son apprentissage de sa langue «maternelle» est d'ailleurs surprenante. Au cours de ses études à Lyon, le Père supérieur lui demande de rédiger un texte en arabe destiné à l'évêque maronite de Beyrouth de passage à Lyon pour lui exposer la formation que dispense le séminaire. Le jeune homme doit alors avouer «qu'il en était bien incapable. L'arabe, il le parlait, le lisait moyennement, l'écrivait un peu, mais pas au point de rédiger un exposé. » (p. 180) L'indignation du Père qui trouve surprenant qu'un Égyptien, destiné à exercer son ministère au Moyen-Orient puisse se permettre de ne pas connaitre convenablement l'arabe, le pousse à réfléchir. Au début de son séjour en France, le jeune homme semble adopter le mode de vie et les pensées de ces condisciples tous issus de la noblesse et de la grande bourgeoisie française, mais cet incident le marque profondément : «André ne songeait plus à ferrailler contre la Révolution française. Il avait remis les pieds sur terre - et en terre égyptienne. Sa manière d'appréhender le monde avait été bouleversée » et il se met alors à une étude sérieuse de l'arabe. Le paradoxe de la situation est souligné avec humour par sa tante Maguy qui dit à Yolande : «Si je comprends bien chérie, (...) ton fils est allé jusqu'à Lyon pour apprendre l'arabe...» (p. 181) Cependant, Edouard Dhellemmes, un industriel lillois venu au Caire pour prospecter le marché égyptien, note lors de son premier voyage au Caire en 1916 que les Égyptiens avaient parfois des expressions bizarres. C'est ce que souligne Jean-Jacques Luthi dans son ouvrage consacré au Français d'Égypte: "On a pu parler d'argot à propos du français d'Égypte en ce sens que les francophones locaux savent bien qu'ils ne peuvent être tout à fait compris que de leur groupe social ou local et, de cefait, leur conversation reste quelquefois obscure pour un Français métropolitain. » (LUTHI, 2005 : 240-241) Lors d'un voyage en France et au cours d'une halte au Café de la Paix, Sélim est confronté à cette incompréhension, quand s'adressant au serveur, il déclare :

Moi, je prendrai une gazeuse avec un chalumeau. (...) Devançant l'interrogation du garçon, Paul explique d'une voix pincée: "Monsieur voudrait un citron pressé avec une paille.» En d'autres circonstances, Sélim aurait éclaté de rire. Mais le ton de son beau-frère le vexa. (p. 299)

Si Sélim est froissé par l'intervention de Paul, c'est qu'il ressent le snobisme sous-jacent. D'origine modeste, son français est le français local que parlent les couches populaires et les remarques de son beau-frère sont en fait destinées à souligner leurs différences sociales.

En effet, les transgressions linguistiques sont plus évidentes dans les milieux populaires et servent souvent à révéler les origines et les appartenances sociales des interlocuteurs. Les amies de Viviane lui laissent entendre que son amoureux n'appartient pas à leur milieu social «il est vrai que Sélim ne s'était pas contenté de multiplier les phrases en arabe: son français, par moments, ressemblait à une traduction. Il leur avait écorché les oreilles en précisant : Je 
travaille comptable chez Matossian.» (p. 260) Cependant, au-delà de ces distinctions, il reste qu'au fil des ans, il s'est développé un parler égyptien bien distinct, un français d'Égypte, à l'image de toute cette population dite d'Égypte, venue de toute part et dont le meilleur symbole était Alexandrie, ville cosmopolite ouverte à toutes les influences et groupant des gens originaires des quatre coins du monde.

Ceci veut-il dire que la langue française fut complètement intériorisée ? Pas nécessairement. Les Égyptiens de souche, comme Salwa, l'amie musulmane de Viviane, ou Makram l'ancien condisciple de Georges, même s'ils maitrisent parfaitement le français, ils ne délaissent pas pour autant l'arabe qu'ils parlent parfaitement bien. En effet, le rapport à la langue française est intimement lié dans ce roman à l'idée que se font les protagonistes de leur identité. Et, ce n'est sans doute pas par hasard, qu'André soit le seul à être resté en Égypte après le départ de toute sa famille. Paul, quant à lui, en guise de rupture définitive avec son passé, refuse, une fois en Suisse, de parler arabe.

En effet, le problème de l'identité ou plutôt des «identités multiples », selon l'heureuse expression d'Amin Maalouf, est au cœur du Tarbouche. D'ailleurs, le projet mémorialiste de l'auteur se prête parfaitement au sujet. D'une part, l'introduction de l'Histoire au sein du récit par le narrateur qui n'a pas connu directement la plupart des événements, établit déjà un certain recul par rapport aux faits; son regard est celui du présent sur le passé. D'autre part, la narration rétrospective, en ressuscitant les expériences passées, conduit à éprouver «ladistance de sa propre altérité dans la représentation d'un devenir différencié. » (RICCEUR, 1996: 175) Cet écart est aussi perceptible dans les passages qui font appel au journal de Michel. La mise en abîme donne lieu à des commentaires et à des interrogations d'ordre identitaire. L'introduction des flux temporel et mémoriel dans le récit fictionnel, fait appel à l'historicité la réminiscence devenant ainsi un vecteur privilégié de la remise en question identitaire.

Dès les premières pages du récit, le narrateur déclare: "On nous appelle les Syriens. Appellation ambiguë qui ignorait le Liban et, surtout, laissait croire que nous appartenions à un autre pays. Comme si nos familles, établies en Égypte depuis des lustres, n'avaient pas définitivement coupé les ponts avec Damas, Alep ou Sidon. »(p. 11) Ceci veut-il dire qu'ils sont syriens? Qu'ils se considèrent comme égyptiens? Ou que ce sont les Égyptiens qui continuent à les regarder comme des intrus? Difficile à dire! La remarque du narrateur ne fait que rendre la situation plus obscure. Pourtant, cette déclaration équivoque s'accorde bien avec la personnalité des protagonistes qui se complaisent dans le flou. Race d'intermédiaire, selon Georges, leur place est toujours entre-deux : «Nous sommes un peuple de commerçants et d'intermédiaires. Nous avons toujours été assis entre deux chaises. C'est parfois inconfortable, mais je crois que nos fesses sont ainsi faites. Tous ceux qui prétendent basculer d'un côté ou de l'autre finissent par se faire très mal. » (p. 130) Le narrateur, plus lucide, et regardant rétrospectivement les faits, reconnaît que c'est surtout pour se différencier des Égyptiens : «Il y avait en Égypte les Anglais, les Grecs, les Italiens, les Arméniens, les Juifs...Nous, nous étions syriens, à défaut d'être égyptiens à part 
entière ou un peu européens.» (p. 11) Cette division communautaire fait partie intégrante de la vie de tous les jours, même pendant les vacances d'été comme nous dit Charles: «Tout le mondese connaissait sur cette plage de Glymenopoulo, colonisée par les Syriens, alors que les Juifs occupaient celle de Stanley toute proche. On se sentait chez soi, entre soi. » (p. 188)

Jusqu'à la chute de l'Empire ottoman, les Syriens étaient des sujets locaux, et non pas des étrangers, ce n'est qu'après le démembrement de ce dernier qu'on commence à se référer juridiquement à eux en tant qu'étrangers ou "personnes à statut mal défini », en attendant la nouvelle loi réglant le problème des nationalités, loi qui tardait bien au dire d'Élias qui, «en 1920, cinquante-neuf ans après son arrivé sur les bords du Nil (...) ne pouvait toujours pas se prévaloir formellement du titre d'Égyptien. » (p. 98) Le traité de Sèvres ne simplifie pas la situation :

Les Syriens d'Égypte devaient choisir entre deux nationalités: celle de leur pays d'origine, passé sous mandat français, ou celle de leur pays d'adoption, devenu protectorat britannique. Pour le moment, ils n'étaient pas surs de posséder ni l'une ni l'autre. L'occupation anglaise puis le démembrement de l'Empire ottoman avaient rendu leur statut plus flou que jamais. (p. 100)

D'ailleurs, comme le souligne Robert Ilbert, historien et spécialiste de l'Égypte, depuis la seconde moitié du XIX ${ }^{\mathrm{e}}$ siècle « on pouvait (...) avoir plusieurs nationalités et jouer d'elles comme de cartes de crédit. » (ILBERT, 1992 : 27) C'est justement ce que tente de faire Georges en 1920 :

Mon grand-père qui, né en Égypte ne se sentait qu'à moitié concerné par le débat. Mais avec sa tendance naturelle à jouer sur plusieurs tableaux et à profiter de toutes les occasions, il se demandait si des égyptianisés comme lui ne pourraient pas obtenir un statut d'étranger à l'occasion des règlements internationaux en cours. (p. 99)

Ce n'est donc pas uniquement par souci d'une étiquette, selon l'expression du narrateur, que les siens maintiennent cette confusion identitaire, mais aussi pour bénéficier et de la protection du gouvernement français et des avantages accordés aux étrangers. Finalement, après avoir bataillé pour rester en Égypte tout en gardant la nationalité syrienne, il finit par préférer avoir la nationalité égyptienne du moment que la nationalité syrienne ou libanaise ne lui ferait pas bénéficier du régime des Capitulations : "Plutôt qu’un demi-étranger, disait-il, je préfère encore être un demi-national. » (p. 130)

En fait, dans cette société cosmopolite, où les Européens jouissent de maints privilèges, les Syriens chrétiens cherchent, dès la fin du XIX ${ }^{\mathrm{e}}$ siècle, à faire prévaloir leur statut de minorité, statut où le religieux et l'ethnique se confondent de façon un peu bizarre. Cette confusion entretenue entre «communauté » d'origine religieuse et «colonie » d'origine nationale est illustrée de façon comique par le narrateur qui relate des discussions familiales à ce propos, discussion qui vient une fois de plus souligner la notion très vague qu'ils se font de leurs origines : 
Pendant toute mon enfance, j'ai entendu les adultes débattre de nos origines avec un luxe d'imprécision (...) Batrakani vient de l'arabe batrak, patriarche. Nous avions certainement un évêque haut placé parmi nos ancêtres.

- Un évêque? Il est fou parole d'honneur! Depuis quand les évêques ont-ils une descendance?

- Vous dites n'importe quoi! criait la tante Nini (...) Nous descendons des croisées, c'est prouvé. (...)

- Tout le monde sait qu'à Damas, dans les registres du patriarcat....

- Je sais - d'ailleurs, c'est prouvé - que les Batrakani ont quitté la Macédoine au seizième siècle (...) pour s'installer en Syrie. Batrakani est un nom grec, comme Sakkakini, Zananiri...

- C'est sans doute pour cette raison qu'on nous appelle grecs-catholiques, concluait la maîtresse de maison en mêlant allégrement l'Église et la géographie. (p. 12)

À la question apparemment simple «Vous êtes égyptiens?» posée par Edouard Dhellemmes, Yolande et Georges donnent des réponses contradictoires :

- Bien sûr! fit Yolande.

La réponse étonna son mari

- Ah bon? D'où par où sommes-nous égyptiens? (p. 28)

Pour Yolande, dont la famille vit en Égypte depuis le XVIII ${ }^{\mathrm{e}}$ siècle, le sentiment d'appartenance est plus fort que pour son mari dont les parents se sont rencontrés sur un bateau de provenance de Beyrouth, et qui garde en mémoire le récit cauchemardesque de sa mère des massacres de Damas en 1860. Au fil des ans, son succès et sa promotion sociale changent son attitude, «il se sentait tout à fait chez lui désormais, intégré et respecté. Égyptien? La question ne se posait même pas.» (p. 160) Il devient de plus en plus lié au pays, ce qui le rapproche d'André : «Un rapprochement entre Georges Batrakani et son fils aîné s'était amorcé à la fin de 1926. Grâce à l'Égypte, en partie (...) Le nouveau bey et le nouvel arabisant s'affichaient égyptiens. » (p. 181) Pourtant, les sentiments de Georges sont plus ambigus que ceux de son fils qui, tout en bataillant contre l'arabisation du collège des Jésuites après l'Affaire de Suez, et tout en poursuivant une lutte acharnée pour garder au français sa place privilégiée au sein de l'institution scolaire, s'affirme en tant qu'Égyptien. D'une part, le nouveau bey se sent de plus en plus d'Égypte et se met en colère quand ses concurrents le traient d'étranger. D'autre part, il prend ses distances avec les gens $\mathrm{du}$ pays et s'identifie aux Européens. Comme eux, il souscrit au mythe de l'Oriental paresseux et malhonnête et se sent supérieur aux indigènes. L'attitude de son fils Paul est plus radicale et sans ambivalence. Européanisé, il cherche, comme beaucoup de Levantins et de Juifs d'origine orientale, à gommer ses origines et à s'assimiler à la culture occidentale. Cette posture est d'ailleurs mise en relief par Edward Saïd qui décrit ces Orientaux évoluant dans un univers occidentalisé qu'ils «considèrent comme le leur, même si ce monde européen du pouvoir et de la domination coloniale les excluait, leur imposait un certain assujettissement et les décevait profondément.» (SAID, 2005 : 349) Invitée avec son mari par le Capitaine Simpson à Guézira, Linda est froissée par l'attitude méprisante de leur hôtesse : «Elle regarda à peine ces Levantins endimanchés et poursuivit sa conversation avec des dames anglaises de son monde. (...) Linda, si fière de sa robe lilas à festons, s'était sentie terriblement 
humiliée par le regard dédaigneux de Mme Simpson. » (p. 54-55) Décidément aussi francophone que son frère, Michel ne cherche pas à nier ses origines, mais il est si imprégné par la culture et par la littérature françaises, qu'il évolue dans un monde à part, un monde réglé par les réunions littéraires qui foisonnent au Caire comme à Alexandrie et où les écrivains «d'expression française » présentent leurs œuvres à un public exigeant et cultivé, un monde où tout se passe exclusivement en français.

Citoyens de seconde zone, au dire de Georges, les Syriens se sentent menacés dès que les choses bougent. Ils ont un désir instinctif de se tenir en retrait. Une fois en exil, Michel reconnait que les siens n'ont pas voulu s'intégrer, préférant s'éloigner et se protéger dans leur cocon des coups et des dangers :

Pour les funérailles du sultan, nous étions au balcon. "C'est de là qu'on voit le mieux», m'avait dit papa (...) Finalement, nous aurons passé la plus grande partie de notre vie au balcon: à regarder passer les autres... Le balcon donnait de la hauteur, mais surtout de la distance; il empêchait de recevoir des coups et de se salir les mains. (p. 412)

Cependant, cet éloignement des réalités du pays s'applique surtout aux nouveaux venus comme Élias et à la classe aisée qui s'assimile plutôt aux Français, comme les amis de Viviane qui sont divisés «en gaullistes et en pétainistes » et qui sont surpris par Sélim quand il déclare à propos de ses opinions politiques : «Moi, c'est l'Égypte qui m'intéresse. » (p. 273)

Par ailleurs, un abîme émotionnel sépare Égyptiens et Syriens. Ceci devient évident dès qu'on aborde la question coloniale. Élias donne un compte rendu enthousiasmé du défilé des troupes britanniques dans les rues du Caire suite à la défaite de l'armée égyptienne :

Quel spectacle, mes enfants! (...) Le défilé des vainqueurs de Tell el Kébir était ouvert par deux drapeaux criblés de balles. Vingt mille hommes suivaient, sans tambour ni clairon. Leur marche silencieuse les rendait plus impressionnants encore. C'était un festival d'uniformes et de couleurs. Entre les vestons écarlates des artilleurs, les pantalons gris perle des fantassins, les jupes plissées des régiments écossais, les costumes bleu indigo et les turbans de la cavalerie indienne, on aurait dit que cinq ou six armées étrangères avaient envahi l'Égypte en même temps. (p. 47)

Makram, pour sa part, est si affecté par l'occupation de son pays qu'il décide de ne porter que du noir en signe de deuil jusqu'au départ des Anglais. Lors des soulèvements populaires réclamant l'indépendance en 1919, Georges se plaint du ralentissement des affaires et n'arrive pas à comprendre les sentiments de son ami d'enfance :

Vous, les Syriens, vous ne pouvez pas comprendre, murmura Makram au bout de trois minutes de silence (...)

- Ah bon, parce que vous, les coptes....

- Qui te parles de coptes? Nous sommes égyptiens. (p. 93)

En effet, Georges ne peut concevoir l'appartenance en d'autres termes que communautaire que ce soit en tant que grec-catholique ou en tant que Syrien, sujet de l'Empire ottoman. Le fort sentiment national qui anime son ami, et qui le pousse à dépasser les différences religieuses et à déclarer : "Quand il s'agit 
de l'Égypte je peux être musulman» (p. 139), ce désir de l'indépendance même au prix de lourds sacrifices qui le travaille, n'est pour Georges, qu'un enfantillage dangereux. Lui, il s'accommode bien de la présence politique britannique doublée de l'hégémonie culturelle et économique françaises, qui arrange bien ses affaires. De même, Viviane est choquée par la réaction de Salwa, son amie musulmane qui, lors de l'affaire de Suez, décide de partir se battre sur le Canal. Les attaques virulentes de son ancienne camarade de classe contre la France la surprennent:

- Qu'est-ce qui te prend? lui demande ma mère avec étonnement. Toi, une ancienne élève de la Mère de Dieu, pétrie de culture française... Tu ne parles que des Français. Il y a aussi des Anglais, que je sache.

- L'Angleterre a toujours été notre ennemi. Mais la France, elle, nous a trahis! (p. 376)

Michel, plus lucide que sa sœur, est soulagé par l'intervention américaine, intervention qui vient empêcher les siens d'être à porte faux une fois de plus :

Michel se disait qu'en 1798, quand Bonaparte avait débarqué en Égypte, les Syriens étaient tombés dans les bras des troupes françaises. Et en 1882, après le soulèvement d'Orabi, ils avaient accueilli l'occupant britannique comme un sauveur. Cette fois, ils se seraient sans doute tenus à plus de réserve, mais auraient-ils vraiment pu cacher leur soulagement si les Américains.... (p. 377)

Gilbert Sinoué, un autre Syrien d'Égypte, se rappelle dans Le Colonel et l'enfant-roi, la réaction ambiguë de son père à l'annonce de l'intervention francobritannique en 1956, intervention qui le satisfait dans la mesure où elle lui laisse entrevoir la chute de Nasser dont la politique nationaliste inquiète, mais qui le navre en même temps :

Chrétiens, Juifs, Grecs, Italiens, dans leur très grande majorité, ces gens installés en Égypte depuis des générations (...) soudain, face à cette attaque, voilà qu'ils se sentent solidaires de l'homme qui, hier encore, était à la source de leurs inquiétudes. À leurs yeux, ce n'est pas Nasser que l'on attaque, mais une terre, la terre de l'Égypte »(SINOUÉ, 2008 : 337)

Puis vient le moment de l'exode ! familles après familles plient leurs bagages et quittent ce pays qui les a vus naitre et grandir. Certains, des Français et des Juifs, sont expulsés pendant «la triple et lâche » comme Edward et Mima ; tandis que d'autres, ne se sentant plus les bienvenus, choisissent de partir : «Non habbibi, personne ne nous a obligés à quitter l'Égypte; mais l'air y devenait irrespirable. Ce n'était plus la même Égypte. Nous sommes partis de notre propre gré, sur la pointe des pieds. » (p. 412) En fait, l'Affaire de Suez marque le début de la fin, la fin de la présence réelle de la France en Égypte, la fin d'un mode de vie, la fin d'une Égypte cosmopolite ouverte où se côtoient toutes les nationalités, tous ses gens dits «d'Égypte». La politique de nationalisation pousse, dès le début des années 60, les hommes d'affaires et industriels à abandonner cet ancien eldorado et à chercher leur chance ailleurs. Les parents du narrateur sont parmi les derniers à s'en aller. Sélim se moque de son frère Jean qui décide de s'installer au Canada, il refuse de croire qu'il n'a plus sa place dans ce pays qu'il considère sien. Contrairement à sa belle-famille très francisée, il a grandi dans un quartier populaire au milieu des Égyptiens, il parle français, mais 
sa langue maternelle est vraiment l'arabe, c'est dans cette langue qu'il exprime le mieux ses émotions. Pourtant, il se sent de plus en plus traqué par Hassan Sabri, un officier au pouvoir et neveu de Rachid, l'ancien Soffragui des Batrakani, il finit lui aussi par partir au Liban. Seul André refuse de partir, quand il va à l'aéroport pour dire au revoir à Viviane et à sa famille, sa sœur s'inquiète pour lui :

- Il n' y aura pratiquement plus de Batrakani en Égypte. Et la plupart des gens que nous connaissons sont partis. Tu vas rester seul...

- Oui, seul avec vingt-huit millions d'habitants, répondit le jésuite d'une voix amusée. (p. 409-410)

Méditant sur la fin tragique de cette communauté si active et si entreprenante et qui, à ses débuts, marqua profondément l'Égypte en servant de trait d'union entre l'Orient et l'Occident et fut un agent indéniable de la modernité du pays, Michel reconnaît qu'André avait raison de dire à Viviane puis à René venu lui annoncer son départ pour le Canada : «Les Syriens d'Égypte se sont trompés. Ils ont cru que la réussite sociale les dispensait d'intégration. (...) Soyez des Canadiens à part entière. Et faites pour le mieux avec les enfants. Car vos racines sont ici et l'arbre va pousser là-bas. » (p. 405-406) Cependant, Michel se dit que la vérité n'est jamais simple surtout dans une histoire à demi teinte comme la leur: «Nous ne sommes pas syriens, nous ne sommes pas égyptiens » avait dit Georges (p. 413), «Nous avons toujours été entre-deux : entre deux langues, entre deux cultures, entre deux Églises, entre deux chaises» reconnait Michel des années plus tard (p. 412), cette situation ambivalente prend toute son ampleur, quand, une fois réfugiés dans la terre de leurs ancêtres, ils se découvrent égyptiens et sont repérés à cent mètres à cause de leur accent :

"Étranges parcours de ceux qu'on appelait Syriens en Égypte, qui se font traiter aujourd'hui d'Égyptiens au Liban et qui se présentent comme Libanais en Europe. » (p. 411)

Histoire de l'ascension et de la dispersion d'une famille, Le Tarbouche est aussi, comme nous venons de le voir, l'histoire d'une certaine Égypte passionnée par la France, et au-delà, l'histoire de toute une époque à jamais révolue.

\section{BIBLIOGRAPHIE}

ABECASSIS Frédéricic (2000), L'enseignement étranger en Égypte et les élites locales 1920-1960, Université d'Aix-Marseille.

Groupe d'études sur l'Islam (1938), L'Égypte indépendante, Paris.

ILBERT Robert (1992), «Une certaine citadinité » dans Alexandrie 1860- 1960, sous la direction de Robert Ilbert et Ilios Yannakakis, Paris, Autrement, Vol. 20.

LUTHI Jean-Jacques (2005), Enquête du Français d'Égypte, Paris, Harmattan.

LUTHI Jean-Jacques (2008), Entretiens avec des auteurs francophones d'Égypte et fragments de correspondances, Paris, Harmattan.

LUTHI Jean-Jacques (2000), L'Égypte des rois, Paris, Harmattan.

RICOEUR Paul (1996), Soi-même comme un autre, Paris, Éditions du Seuil.

SAID Edward (2005), Culture et impérialisme, Paris, Fayard. 
SINOUÉ Gilbert (2008), Le colonel et l'enfant-roi, Paris, Gallimard, collection Folio.

SolÉ Robert (1992), Le Tarbouche, Paris, Éditions du Seuil.

SolÉ Robert (1997), L'Égypte, passion française, Paris, Editions du Seuil. 